

\title{
First Measurement of the Giant Monopole and Quadrupole Resonances in a Short-Lived Nucleus: $56 \mathrm{Ni}$
}

C. Monrozeau, E. Khan, Y. Blumenfeld, C. E. Demonchy, W. Mittig, P. Roussel-Chomaz, D. Beaumel, M. Caamaño, D. Cortina-Gil, J. P. Ebran, et al.

\section{- To cite this version:}

C. Monrozeau, E. Khan, Y. Blumenfeld, C. E. Demonchy, W. Mittig, et al.. First Measurement of the Giant Monopole and Quadrupole Resonances in a Short-Lived Nucleus: 56Ni. Physical Review Letters, 2008, 100, pp.042501. 10.1103/PhysRevLett.100.042501 . in2p3-00238507

\section{HAL Id: in2p3-00238507 https://hal.in2p3.fr/in2p3-00238507}

Submitted on 22 Feb 2008

HAL is a multi-disciplinary open access archive for the deposit and dissemination of scientific research documents, whether they are published or not. The documents may come from teaching and research institutions in France or abroad, or from public or private research centers.
L'archive ouverte pluridisciplinaire HAL, est destinée au dépôt et à la diffusion de documents scientifiques de niveau recherche, publiés ou non, émanant des établissements d'enseignement et de recherche français ou étrangers, des laboratoires publics ou privés. 


\title{
First Measurement of the Giant Monopole and Quadrupole Resonances in a Short-Lived Nucleus: ${ }^{56} \mathrm{Ni}$
}

\author{
C. Monrozeau, ${ }^{1}$ E. Khan, ${ }^{1}$ Y. Blumenfeld, ${ }^{1}$ C.E. Demonchy,${ }^{2}$ W. Mittig, ${ }^{3}$ P. Roussel-Chomaz, ${ }^{3}$ \\ D. Beaumel, ${ }^{1}$ M. Caamaño, ${ }^{4}$ D. Cortina-Gil, ${ }^{4}$ J.P. Ebran,${ }^{1}$ N. Frascaria, ${ }^{1}$ U. Garg, ${ }^{5}$ M. Gelin, ${ }^{3}$

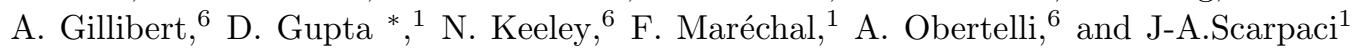 \\ ${ }^{1}$ Institut de Physique Nucléaire IN2P3/CNRS, Univ. Paris Sud, 91406 Orsay Cedex, France \\ ${ }^{2}$ Univ. of Liverpool, Dep. of Physics, Olivier Lodge Lab., Liverpool L69 7ZE, U.K. \\ ${ }^{3}$ GANIL, DSM/CEA, IN2P3/CNRS, BP 5027, 14076 Caen Cedex 5, France \\ ${ }^{4}$ Univ. Santiago de Compostela, E-15706 Santiago de Compostela, Spain \\ ${ }^{5}$ Physics Department, University of Notre Dame, Notre Dame, IN 46556, USA \\ ${ }^{6}$ CEA/DSM/DAPNIA/SPhN, Saclay, 91191 Gif-sur-Yvette Cedex, France
}

\begin{abstract}
The Isoscalar Giant Monopole Resonance (GMR) and Giant Quadrupole Resonance (GQR) have been measured in the ${ }^{56} \mathrm{Ni}$ unstable nucleus by inducing the ${ }^{56} \mathrm{Ni}(\mathrm{d}$, d') reaction at $50 \mathrm{~A} . \mathrm{MeV}$ in the Maya active target at the GANIL facility. The GMR and GQR centroids are measured at $19.3 \pm$ $0.5 \mathrm{MeV}$ and $16.2 \pm 0.5 \mathrm{MeV}$, respectively. The corresponding angular distributions are extracted from 3 to 7 degrees. A multipole decomposition analysis using Distorted Wave Born Approximation with Random Phase Approximation transition densities shows that both the GMR and the GQR exhaust a large fraction of the energy-weighted sum rule. The demonstration of this new method opens a broad range of giant resonance studies at intermediate energy radioactive beam facilities.
\end{abstract}

PACS numbers: 21.10.Re,24.30.Cz,21.60.Jz,24.50.+g,21.60.Ev

Over the past few years the focus of nuclear structure has moved towards short-lived exotic nuclei. Major discoveries of unexpected new phenomena have been recorded, such as neutron haloes and skins [1] and the modification of magic numbers far from stability [2]. Giant resonances are collective excitations of nuclei involving a large fraction of the constituent nucleons. They have proved to be a major source of information on collective and single-particle properties of atomic nuclei [3]. The reproduction of their properties represents a strong constraint for nuclear models, in particular modern microscopic approaches based on the density functional theory [4]. Fundamental nuclear properties such as the equation of state of nuclear matter or the effective nucleonnucleon interaction have benefited from detailed measurements of giant resonances (GR) [5]. The study of GR's in exotic nuclei is thus expected to enhance our understanding of exotic nuclear structure. Experimentally such studies encounter major hurdles and have until now been limited to the isovector giant dipole resonance in neutron-rich Oxygen [6], Neon [7] and Tin isotopes [8]. They provided the first evidence for soft E1 modes in unstable nuclei.

The isoscalar Giant Monopole Resonance (GMR) is of major importance because its properties are related to the nuclear matter incompressibility $K_{\infty}$. The interest in determining $K_{\infty}$ stems from its impact on the nuclear matter equation of state. Some significant progress in our

* Present address: Variable Energy Cyclotron Centre, 1/AF Bidhan Nagar, Kolkata 700064, India understanding of how $K_{\infty}$ can be constrained has been achieved in recent times. Namely, the procedure generally adopted to extract the incompressibility from the measurement of the GMR energy [9] is based on energy functionals which allow one to calculate finite nuclei and nuclear matter on the same footing [10]. The first step consists in constraining the energy functional in order to reproduce the experimental value of the monopole energy in a given nucleus $[11,12]$. Then using this constrained energy functional, the value of $K_{\infty}$ is extracted.

Within the relativistic and non-relativistic frameworks, $K_{\infty}$ can be fixed with an accuracy of $\sim 10 \%$ [12]: $K_{\infty} \approx$ $230-250 \mathrm{MeV}$. Part of the uncertainty is due to our poor knowledge of the symmetry energy [12]. Therefore, the investigation of the GMR along isotopic chains should be relevant to probe the role played by the symmetry energy in the determination of the nuclear matter incompressibility. Moreover, the incompressibility of asymmetric matter is a basic parameter in calculations describing neutron stars or supernovae [13]. Measurement of the GMR in neutron-rich systems may be a milestone towards testing the energy functionals to predict the equation of state in asymmetric infinite matter.

It is therefore tantalizing to achieve a pioneering method to measure the GMR in unstable nuclei. The ${ }^{56} \mathrm{Ni}$ nucleus plays a major role in stellar nucleosynthesis: it was observed in the ejecta from supernova 1987A [14] and is related to the so-called iron peak. This provides strong motivation to investigate the structure of this nucleus, as stressed in Ref. [15]. The measurement of GMR and GQR in ${ }^{56} \mathrm{Ni}$ also represents a first step in the study along the $\mathrm{Ni}$ isotopic chain.

The best probes for the investigation of the GMR and 
the Giant Quadrupole Resonance (GQR) are isoscalar probes such as deuterons or alpha particles at energies between a few tens and a few hundreds A.MeV [3]. No such experiment has been performed for unstable nuclei up to now, due to the difficult conditions in reverse kinematics. Indeed, the GMR cross section peaks at 0 deg. in the center of mass frame which gives rise to very low recoil velocities for the light probe. To measure the excitation energy range between 0 to $30 \mathrm{MeV}$ in reverse kinematics, it is necessary to detect the recoiling particle ( $\mathrm{d}$ or $\alpha$ ) with energies ranging from $100 \mathrm{keV}$ to $2 \mathrm{MeV}$ at angles from 0 to 40 degrees in the laboratory frame. A standard set-up with a recoiling particle telescope would necessitate a very thin target $\left(\sim 100 \mu \mathrm{g} / \mathrm{cm}^{2}\right)$ to minimize straggling and thus require an intensity of over $10^{7}$ pps which is prohibitive for current radioactive beam facilities.

With respect to the above experimental constraints an active target such as Maya [16] is the key to measuring the GMR and GQR in unstable nuclei. In an active target, the detector gas also acts as target. Such a set-up has in principle an angular coverage close to $4 \pi$, a low energy threshold and a large effective target thickness. In this Letter we report on the first measurement of the GMR and the GQR with a radioactive beam using this new experimental technique.

In the domain of secondary beams, the active target archetype is the detector IKAR [17] which was used at the GSI facility. Maya, developed at GANIL for the lowerenergy domain [16], can be characterized as a $28 \times 26 \times 20$ $\mathrm{cm}^{3}$ Time and Charge Projection Chamber. The electrons from the ionization of the gas by particles drift down the electric field to amplifying wires set parallel to the beam. For a two body reaction, scattered and recoiling particles are in a plane which can be determined by the drift time to the wires. The amplified signal is induced on the anode, a matrix of 35 by 34 pads connected to Gassiplex [18] chips. A hexagonal structure was chosen for these pads in order to have the best conditions for the reconstruction of the projected recoil trajectory, independent of the recoil direction.

The secondary ${ }^{56} \mathrm{Ni}$ beam at $50 \mathrm{~A} . \mathrm{MeV}( \pm 0.5 \%$ energy spread) was produced at the GANIL facility by fragmentation of ${ }^{58} \mathrm{Ni}$ at $75 \mathrm{~A} . \mathrm{MeV}$ on a $70.5 \mathrm{mg} / \mathrm{cm}^{2} \mathrm{C}$ target located in the SISSI [19] device and purified by passing through a $135 \mathrm{mg} / \mathrm{cm}^{2} \mathrm{Al}$ degrader placed between the two dipoles of the alpha fragment separator before being sent to the SPEG area. Maya was placed on the focal plane of the SPEG spectrometer [20] which was used to purify the beam, so only ${ }^{56} \mathrm{Ni}^{27+}$ was transmitted. Two plates were added above and below the beam trajectory in Maya to prevent the highly ionizing beam particles from inducing charges. However, due to the angular spread of the beam, some non-interacting ${ }^{56} \mathrm{Ni}$ ions were detected. This limited the beam intensity that could be used to $5.10^{4} \mathrm{pps}$, while $10^{6} \mathrm{pps}$ of ${ }^{56} \mathrm{Ni}$ were available.

The Maya active target was filled with deuterium gas, at a pressure of $1050 \mathrm{mbar}$, forming a pure deuterium target of $1.6 \mathrm{mg} / \mathrm{cm}^{2}$ (equivalent to a target of $6.3 \mathrm{mg} / \mathrm{cm}^{2}$ of $\mathrm{CD}_{2}$ ). It should be noted that alpha particle scattering, which would be the preferred probe for the GMR, could not be undertaken because the detector, like any gaseous detector, sparked when filled with pure He. As pointed out above, reverse kinematics generate recoil particles in a large energy domain. High energy light particles such as deuterons with $E \geq 2 \mathrm{MeV}$ were not stopped in the Maya gas volume. For such escaping particles, we added ancillary $500 \mu \mathrm{m}$ Si detectors outside the active volume covering from 10 to 60 degrees around the beam direction for events occurring at the center of the detector. For normalization purposes the beam was counted in a polycrystalline diamond detector of $1 \mathrm{~cm}^{2}$ surface and $100 \mu \mathrm{m}$ thickness, after traversing Maya.

For each event two physical observables are determined in order to reconstruct the kinematics of the reaction : the energy and the angle of the recoil. The recoiling angle in the horizontal plane is reconstructed by a linear least squares fit of the positions of the pads hit with a weight proportional to the amount of charge deposited. In order to obtain a reliable trajectory, at least 10 pads with non-zero charges were required corresponding to $5 \mathrm{~cm}$ long trajectories, which introduced an effective deuteron threshold of $700 \mathrm{keV}$. The reaction plane is determined from the drift times of the electrons towards the anode wires. The intersection of the deuteron trajectory with the beam axis gives the vertex of the interaction. The recoil stopping point is determined by projecting the charges on the direction of the trajectory whose maximum corresponds to the Bragg peak. For each event, the recoil angle and the range in the deuterium gas are therefore determined. The energy of the recoil is then deduced from its range in the gas through energy-loss tables [21]. The 3D-reconstruction of scattering trajectories in Maya provides the kinematics of the ${ }^{56} \mathrm{Ni}\left(\mathrm{d}, \mathrm{d}^{\prime}\right)$ reaction, shown in figure 1 . The decrease of intensity to small recoiling deuteron angles is mainly due to the shielding of the beam, leading to an acceptance cut.

The data are then transformed into the center of mass frame using two-body relativistic kinematics. The corresponding excitation energy spectrum of ${ }^{56} \mathrm{Ni}$ corrected for the geometrical efficiency of the detector, is shown in figure 1. This spectrum presents a peak centered at $0 \mathrm{MeV}$ corresponding to elastic and inelastic scattering towards low-lying states. It must be noted that due to the accessible deuteron energy range, the elastic scattering is measured over only a very small angular domain in the center of mass frame, precluding the extraction of the corresponding angular distribution. For the elastic peak the FWHM is $3 \mathrm{MeV}$ and is mainly induced by the trajectory reconstruction uncertainties, especially the vertex localisation due to the beam shielding. At energies ranging from 12 to $25 \mathrm{MeV}$ a bump in the excitation energy spectrum points to the presence of isoscalar giant resonances. Only deuterons corresponding to excitation energy below $25 \mathrm{MeV}$ are stopped in Maya, whereas the 

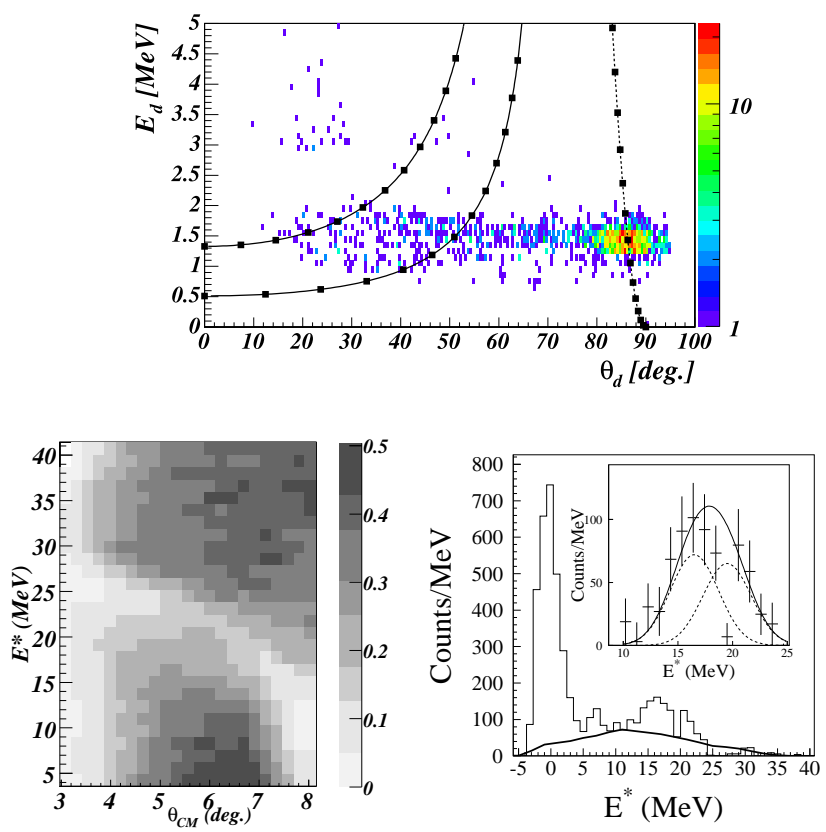

FIG. 1: Top (color online): Scatterplot of recoiling deuteron energy versus scattering angle in the laboratory frame for the ${ }^{56} \mathrm{Ni}$ beam. The lines correspond to elastic scattering and the $14 \mathrm{MeV}-22 \mathrm{MeV}$ excitation energy of ${ }^{56} \mathrm{Ni}$. The CM angles ares denoted by squares along these lines, with $1 \mathrm{deg}$. step, starting from $0 \mathrm{deg}$. for the lowest deuteron energy. Bottom left: geometrical efficiency of the detection setup in the $\mathrm{CM}$ frame. Bottom right: ${ }^{56} \mathrm{Ni}$ excitation energy spectrum deduced from the deuteron kinematics and corrected for geometrical efficiency. The background which was subtracted is shown by the solid line. The insert shows the background subtracted inelastic data fitted with Gaussians located at 16.5 $\mathrm{MeV}$ and $19.5 \mathrm{MeV}$ for the GQR and the GMR, respectively (see text).

Si detectors placed $70 \mathrm{~mm}$ behind the Maya detector allow the energy spectrum between $28 \mathrm{MeV}$ and $35 \mathrm{MeV}$ to be measured.

Since the pressure and the bias used in the experiment were optimized to detect very low deuteron energies, it was not possible to separate protons from deuterons through range vs. charge measurements [16]. Consequently some proton contamination may be expected in the data. Protons can be produced essentially by two mechanisms : deuteron break-up and one neutron transfer $(\mathrm{d}, \mathrm{p})$ reactions. The latter will not be considered since the lowest energy protons are scattered at backward angles. The contribution of the deuteron break-up was estimated from direct kinematic measurements for ${ }^{58} \mathrm{Ni}$ at 50 A.MeV at larger angles $\left(\theta_{C M}>8\right.$ deg.) [22]. These data are extrapolated to lower angles and transformed to the reverse kinematics frame, corrected for the geometrical acceptance of Maya and normalized to the experimental data (Fig. 1). The shape of the deuteron breakup does not exhibit any bump in the giant resonance region but rather corresponds to a smooth background.



FIG. 2: GMR and GQR inelastic angular distributions of ${ }^{56} \mathrm{Ni}$ at $50 \mathrm{~A} \cdot \mathrm{MeV}$ and DWBA calculations using microscopic double folded RPA form factor (see text). The data (squares) are also displayed. Bottom right: analysis of the data obtained by using two Gaussians located at $16.5 \mathrm{MeV}$ and $19.5 \mathrm{MeV}$ for the GQR and the GMR, respectively, with FWHM=5.2 $\mathrm{MeV}$ (see text).

In order to extract the GMR and GQR centroid location and the corresponding angular distributions, a Multipole Decomposition Analysis (MDA) is performed [3]. After subtraction of the background, experimental angular distributions were extracted for five excitation energy intervals and fitted by a linear combination of theoretical distributions for $\mathrm{L}=0$ and $\mathrm{L}=2$ excitations. The theoretical angular distributions are calculated using the Distorted Wave Born Approximation (DWBA). Both real and imaginary optical potentials are microscopically determined using ${ }^{56} \mathrm{Ni}$ and deuteron densities within the double folding model with the M3Y interaction as in [23]. The deuteron density is obtained from the Hulthen deuteron wave function [24]. The ${ }^{56} \mathrm{Ni}$ nucleus densities are calculated with the Hartree-Fock model and the SGII $\left(K_{\infty}=217 \mathrm{MeV}\right)$ [5] Skyrme functional without including pairing since it is a doubly magic nucleus. The corresponding $\mathrm{L}=0$ and $\mathrm{L}=2$ strength functions are well concentrated, similar to those of Fig. 3 and 28, respectively, 
of Ref. [25]. No data are available for deuteron elastic scattering from the unstable ${ }^{56} \mathrm{Ni}$ nucleus therefore the optical potentials are renormalized in order to reproduce ${ }^{58} \mathrm{Ni}(\mathrm{d}, \mathrm{d})$ at $120 \mathrm{MeV}[26]$ : the real part of the optical potential is increased by $7 \%$. The GMR and GQR transition potentials are calculated with the same procedure, using microscopic RPA transition densities [27] obtained self-consistently from the same Skyrme functional as in the HF calculation. It should be noted that it is the first time that the GMR is measured for such a light nucleus using the $\left(\mathrm{d}, \mathrm{d}^{\prime}\right)$ reaction. Consequently, the validity of reaction models cannot be thoroughly tested on stable nuclei for this reaction.

Fig. 2 shows the angular distributions whose shapes are well reproduced by the calculations. As expected, the $\mathrm{L}=2$ mode dominates below $17 \mathrm{MeV}$ whereas the $\mathrm{L}=0$ mode is the largest above $17 \mathrm{MeV}$. The percentage of the exhausted Energy-Weighted Sum Rule (EWSR) is determined for each energy interval for both multipolarities. It is then straightforward to obtain the centroid of the giant resonances and the total exhausted EWSR (Table I). Error bars are purely statistical and do not take into account uncertainties on the background or on the reaction models. In order to cross-check the results, another independent analysis of the data was undertaken by fitting the excitation energy spectrum with the background shape and two Gaussians corresponding to the GMR and the GQR, for different angles between $3 \mathrm{deg}$. and $7 \mathrm{deg}$. The angular distributions of the Gaussians are shown in the bottom right part of Fig. 2 and are well reproduced by DWBA calculations. The percentage of EWSR extracted by normalisation are in close agreement with the MDA results.

The ${ }^{56} \mathrm{Ni}$ results are comparable to those obtained on ${ }^{58} \mathrm{Ni}$ and ${ }^{56} \mathrm{Fe}$ with $\left(\alpha, \alpha^{\prime}\right)$ scattering $[28,29]$ (Table I).
The FWHM obtained by the Gaussian fits on the ${ }^{56} \mathrm{Ni}$ data $(5.2 \pm 0.5 \mathrm{MeV}$ both for the GMR and the GQR) are also consistent with neighbouring stable nuclei where FWHM between $5.5 \mathrm{MeV}$ and $7.7 \mathrm{MeV}$ are found [28].

\begin{tabular}{|c||c|c||c|c|}
\hline \multicolumn{1}{|c||}{} & \multicolumn{2}{c||}{ GMR } & \multicolumn{2}{c|}{ GQR } \\
\cline { 2 - 5 } & $\mathrm{E}_{c}(\mathrm{MeV})$ & $\%$ EWSR & $\mathrm{E}_{c}(\mathrm{MeV})$ & $\%$ EWSR \\
\hline${ }^{56} \mathrm{Ni}$ & $19.3 \pm 0.5$ & $136 \pm 27$ & $16.2 \pm 0.5$ & $76 \pm 13$ \\
\hline${ }^{58} \mathrm{Ni}[28]$ & $19.20_{-0.19}^{+0.44}$ & $85_{-10}^{+13}$ & $16.31_{-0.10}^{+0.17}$ & $82 \pm 10$ \\
\hline${ }^{58} \mathrm{Ni}[29]$ & $19.9_{-0.8}^{+0.7}$ & $92_{-3}^{+4}$ & $16.3_{-0.9}^{+0.8}$ & $73 \pm 3$ \\
\hline${ }^{56} \mathrm{Fe}[28]$ & $18.35_{-0.19}^{+0.33}$ & $98_{-10}^{+14}$ & $16.35_{-0.10}^{+0.19}$ & $77 \pm 10$ \\
\hline
\end{tabular}

TABLE I:

GMR and GQR centroids and EWSR measured in ${ }^{56} \mathrm{Ni}$,

$$
{ }^{58} \mathrm{Ni} \text { and }{ }^{56} \mathrm{Fe}
$$

With only 15 hours of effective data taking and an average beam intensity of $5.10^{4} \mathrm{pps}$, isoscalar GMR and GQR resonances were measured in the ${ }^{56} \mathrm{Ni}$ unstable nucleus. It is the first measurement of these resonances in a short-lived nucleus. This makes the method relevant with respect to predicted production rates of a large domain of exotic nuclei at current and future facilities. A redesign of the detector in order to increase the amplification gain would allow to access to smaller angle measurements and improve particle identification. Measurements in neutron-rich Nickel isotopes up to ${ }^{68} \mathrm{Ni}$ could be undertaken as of now in order to probe the evolution of giant resonances along an isotopic chain. Also, measurements on heavier nuclei such as ${ }^{132} \mathrm{Sn}$ would complement the systematics already existing for stable tin isotopes [30].

This work has been supported in part by CNRS/IN2P3, CEA/DAPNIA and by the U.S. National Science Foundation (Grant No. PHY04-57120).
[1] A. S. Jensen, K. Riisager, and D. V. Fedorov, Rev. Mod. Phys. 76 (2004) 215

[2] T. Otsuka et al., Phys. Rev. Lett. 97 (2006) 162501

[3] M. Harakeh, A. Van der Woude, Giant Resonances, Oxford University Press (2001)

[4] W. Kohn, Rev. Mod. Phys. 71 (1999) 1253

[5] Nguyen Van Giai and H. Sagawa, Nucl. Phys. A371 (1981) 1.

[6] A. Leistenschneider et al., Phys. Rev. Lett. 86 (2001) 5542

[7] J. Gibelin et al., Nucl. Phys. A788 (2007) 153c

[8] P. Adrich et al., Phys. Rev. Lett. 95 (2005) 132501

[9] D.H. Youngblood et al., Phys. Rev. Lett. 39 (1977) 1188

[10] J.P. Blaizot, Phys. Rep. 64 (1980) 171

[11] O. Bohigas, A.M. Lane, J. Martorell, Phys. Rep. 52, (1979) 267

[12] G. Colò et al., Phys. Rev. C70 (2004) 024307

[13] N. K. Glendenning, Compact Stars, Springer-Verlag, N. Y. (1997)

[14] H.A. Bethe, Rev. Mod. Phys. 62 (1990) 801

[15] G. Kraus et al., Phys. Rev. Lett. 73 (1994) 1773
[16] C.E. Demonchy et al., Nucl. Instr. and Meth. A573 (2007) 145

[17] A.A. Vorobyov et al., Nucl. Inst. Meth. 119 (1974) 509 ; Nucl. Inst. Meth. A270 (1988) 419

[18] J. C. Santiard et al., Report CERN-ECP-94-17 (1994).

[19] R. Anne, Nucl. Instr. and Meth. B126 (1997) 279

[20] L. Bianchi et al., Nucl. Instr. and Meth. A276 (1989) 509

[21] L.C. Northcliffe and R.F. Schilling, Nucl. Data Tables A7 (1970) 322

[22] D.Ridikas et al., Phys. Rev. C63 (2000) 014610

[23] J. Cook, Nucl. Phys. A382 (1982) 71

[24] L. Hulthén and M. Sugarawa, The two-nucleons Problem, Springer-Verlag, Berlin (1957)

[25] J. Terasaki and J. Engel, Phys. Rev. C74 (2006) 044301

[26] A.C. Betker et al., Phys. Rev. C48 (1993) 2085

[27] P. Chomaz et al., Phys. Lett. B281 (1992) 6

[28] Y.-W. Lui et al., Phys. Rev. C73 (2006) 014314

[29] B.K. Nayak et al., Phys. Lett. B637 (2006) 43

[30] T. Li et al., Phys. Rev. Lett. 99 (2007) 162503 